\section{$T$ (Brachyury) is a direct target of Wnt3a during paraxial mesoderm specification}

\author{
Terry P. Yamaguchi, ${ }^{1-3}$ Shinji Takada, ${ }^{3-5}$ \\ Yoshiaki Yoshikawa, ${ }^{4,6}$ Nongying $\mathrm{Wu}^{1}{ }^{1}$ \\ and Andrew P. McMahon ${ }^{1,7}$
}

\begin{abstract}
${ }^{1}$ Department of Molecular and Cellular Biology, Biological Laboratories, Harvard University, Cambridge, Massachusetts 02138 USA; ${ }^{4}$ Center for Molecular and Developmental Biology, Graduate School of Science, Kyoto University, Kitashirakawa, Sakyo-ku, Kyoto 606-8502, Japan; ${ }^{5}$ Kondoh Differentiation Signaling Project, Exploratory Research for Advanced Technology (ERATO), Japan Science and Technology Corporation (JST), Sakyo-ku, Kyoto 606-8305, Japan; ${ }^{6}$ Department of Dermatology, Graduate School of Medicine, Kyoto University, Sakyo-ku, Kyoto 606-8501, Japan
\end{abstract}

Wnt3a encodes a signal that is expressed in the primitive streak of the gastrulating mouse embryo and is required for paraxial mesoderm development. In its absence cells adopt ectopic neural fates. Embryos lacking the T-boxcontaining transcription factors, Brachyury or Tbx6, also lack paraxial mesoderm. Here we show that Brachyury is specifically down-regulated in Wnt $3 a$ mutants in cells fated to form paraxial mesoderm. Transgenic analysis of the $T$ promoter identifies $T$ (Brachyury) as a direct transcriptional target of the Wnt signaling pathway. Our results suggest that Wnt3a, signaling via Brachyury, modulates a balance between mesodermal and neural cell fates during gastrulation.

Received September 13, 1999; revised version accepted October 13, 1999.

The embryonic mesoderm of the mammalian embryo is formed by a series of inductive interactions first in the primitive streak, which gives rise to head and trunk mesoderm, and later in the tailbud, which generates the most posterior mesoderm of the tail. As development progresses, successively posterior structures are generated, leading to a posterior extension of the body axis. Previous studies have established that Wnt3a, which encodes a member of the Wnt family of secreted signaling molecules (for review, see Cadigan and Nusse 1997; Moon et al. 1997), is expressed in pluripotent ectoderm cells of the primitive streak during gastrulation (Takada et al. 1994). At early somite stages [8.0-8.5 days postcoitum (dpc)], the Wnt3a expression domain correlates with a domain of cells in the anterior primitive streak fated to give rise to paraxial mesoderm (for review, see Tam and

[Key Words: Gastrulation; mesoderm; neural; cell fate; Wnt; Brachyury] ${ }^{2}$ Present address as of 1/1/2000: Cancer and Developmental Biology Laboratory, National Cancer Institute-Frederick Cancer Research and Development Center, Frederick, Maryland 27102 USA.

${ }^{3}$ These authors contributed equally.

${ }^{7}$ Corresponding author.

E-MAIL amcmahon@biosun.harvard.edu; FAX (617) 496-3763.
Trainor 1994; Wilson and Beddington 1996). Moreover, the anterior and lateral limits of the Wnt3a expression domain lie between cells fated to give rise to paraxial mesoderm and cells that will give rise to neural ectoderm.

A requirement for Wnt $3 a$ in the specification of trunk and tail paraxial somitic mesoderm fates has been demonstrated by mutant analyses. Wnt $3 a$ homozygous null mutant embryos lack all but the anterior-most seven to nine somites (Takada et al. 1994; Greco et al. 1996; Yoshikawa et al. 1997). As a consequence, only the most rostral cervical vertebrae are formed. Histological and molecular analyses demonstrate that ectopic neural structures form in place of posterior paraxial mesoderm (Yoshikawa et al. 1997). Similar results have been reported recently for compound mutants in the high mobility group (HMG) domain containing transcriptional regulators Lef1 and Tcf1 (Galceran et al. 1999). Because members of this gene family function as transcriptional effectors of Wnt signaling (Nusse 1999), these results indicate that Lef1 and Tcf1 likely mediate Wnt3a's effects on paraxial mesoderm development.

Mesoderm specification is thought to be regulated, at least in part, by members of the T-box gene family of DNA-binding transcription factors (Smith 1999). Two of these, $T$ and $T b \times 6$, are coexpressed with Wnt $3 a$ in the primitive streak during gastrulation (Takada et al. 1994; Chapman et al. 1996). Mutations in either gene lead to a loss of trunk and tail mesoderm (Chesley 1935; Chapman and Papaioannou 1998). Ectopic neural tubes form in place of paraxial mesoderm in the Tbx6 mutants, but it is not clear how similar the neural tube abnormalities noted in the Thomozygotes are to the Wnt3a phenotype. Given the similarities between the Wnt $3 a$ and T-box mutant phenotypes, we have investigated the possibility that Wnt3a signaling functions to regulate T-box activity.

\section{Results and Discussion}

To begin to unravel the potential relationships between Wnt $3 a, T$, and $T b \times 6$, we first examined the extent of the similarities between the Wnt3a and T mutant neural tube phenotypes. Histological analysis of serial cross sections through the posterior neural folds of an $8.0 \mathrm{dpc} T$ homozygous mutant demonstrated abnormal folding and kinking of the neural folds that culminated in tubular epithelial structures lying ventral to the developing endogenous neural folds (data not shown). Whole-mount in situ hybridization analysis of $9.5 \mathrm{dpc} T$ mutants for the expression of genes that mark the neural or mesoderm lineages reveals the neural character of the ectopic tubes. Although the expression of markers of paraxial mesoderm such as Mox1 (Fig. 1A) are significantly reduced in the posterior end of the $T$ mutant embryo as expected (Fig. 1B), neural tube markers such as the dorsally expressed Wnt3a (Fig. 1C) and Hes5 (Fig. 1E) are expressed ectopically (Fig. 1D and F, respectively). Examination of 

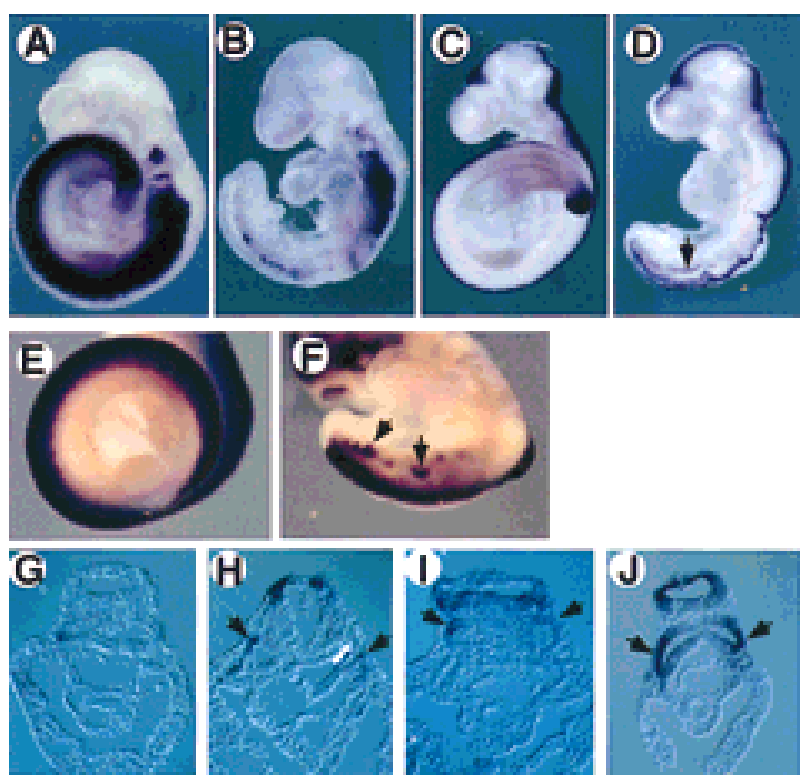

Figure 1. Expression of mesodermal and neural markers indicates ectopic neural tube formation in $T / T$ mutant embryos. Wild-type $(A, C, E)$ and $T / T$ mutant $(B, D, F, G, H, I, J)$ embryos were hybridized with Mox1 $(A, B, G)$, Wnt3a $(C, D, H)$, Hes5 $(E, F, I)$, and Pax3 (J) probes. Whole-mount embryos $(A-F)$ and transverse sections posterior to the forelimb level $(G-J)$ are depicted. In a $T / T$ mutant embryo, several neural markers including Wnt3a, Hes5, and $P a x 3$ are expressed in an ectopic tube lying ventral to the endogenous neural tube (arrows in $D, F, H, I, J$ ) demonstrating the neural character of the ectopic tube.

sections of whole-mount stained mutant embryos demonstrates ectopic expression of Wnt3a (Fig. 1H), Hes5 (Fig. 1I), and Pax3 (Fig. 1J) in ectopic tubular epithelial structures that lie immediately ventral to the normal neural tube. Thus, as in Wnt3a null mutants (Takada et al. 1994; Yoshikawa et al. 1997), ectopic neural structures appear in embryos homozygous for the $T$ mutation. Interestingly, small patches of Mox1 expression are observed occasionally in the ectopic tubes (Fig. 1B,G) suggesting that some cells may still maintain mesodermal character. Taken together, these results demonstrate the presence of ectopic neural tube-like structures in regions displaying reduced paraxial mesoderm, further emphasizing the similarities between the $T, T b \times 6$, and Wnt3a mutant phenotypes.

To determine whether $T$ or $T b x 6$ could function in the Wnt $3 a$ signaling pathway, we examined their expression in embryos lacking Wnt $3 a$ function. Activation of $T$ and Tbx6 transcription is independent of Wnt $3 a$, as both genes are expressed prior to the onset of Wnt $3 a$ expression at 7.5 dpc (Wilkinson et al. 1990; Takada et al. 1994; Chapman et al. 1996). However, Wnt3a could be required to maintain their transcription. Because the first 7 somites form in $T, T b \times 6$, and Wnt $3 a$ homozygous mutants, and the precursors of the more posterior somites 8-12 leave the streak at the 3- to 7-somite stage (Wilson and Beddington 1996; Tam and Beddington 1987), we reasoned that examination of $T$ and $T b \times 6$ gene expression in Wnt3a mutant embryos should focus on these early somite stages. At this time, $T$ mRNA is normally expressed throughout the entire anterior-posterior (A-P) length of the primitive streak, as well as the node and notochord (Fig. 2A,B). However, $T$ expression was completely absent from the anterior half of the primitive streak of 2- to 4-somite Wnt3a $a^{-/-}$embryos (arrows, Fig. 2C,D). The down-regulation of $T$ in the anterior streak was observed as early as the 0 -somite stage (data not shown), several hours before any morphological abnormalities were evident in the mutant. The remainder of the $T$ expression domain in the posterior streak, node, and notochord was unaffected at these stages except for an abnormal domain of $T$ expression in the posterior region of the node (data not shown). Thus, the specific

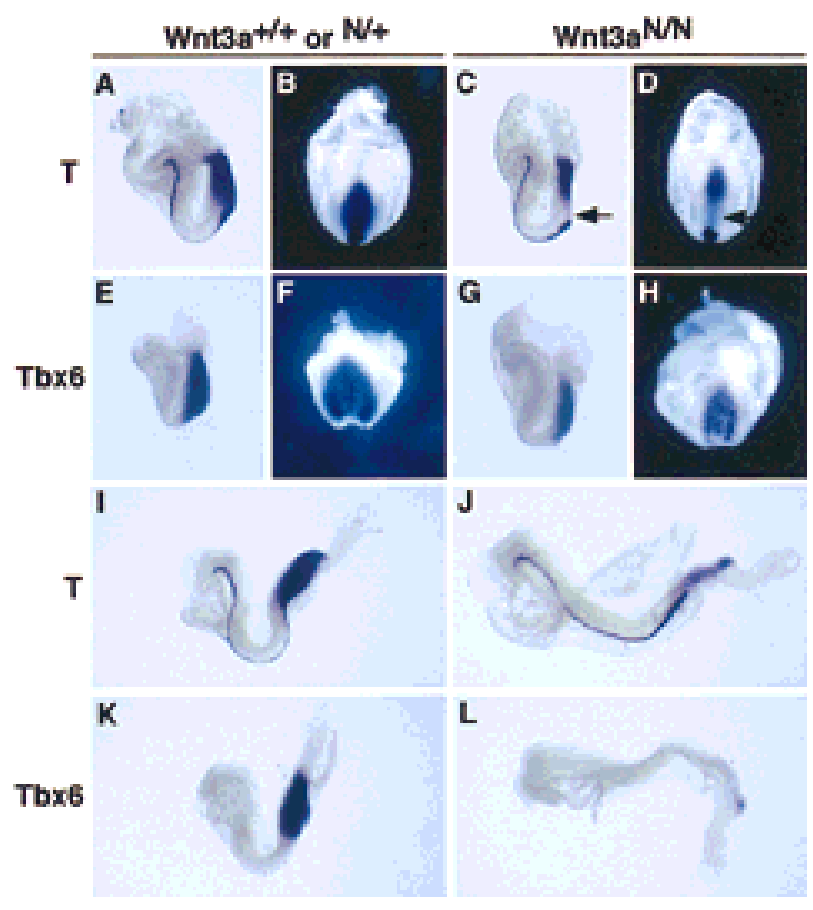

Figure 2. In situ hybridization analysis of T-box gene expression in early somite-stage embryos lacking Wnt3a activity. Embryos are viewed laterally and oriented such that anterior is to the left and dorsal is up, with the exception of $B, D, F$, and $H$, which are ventral-posterior views. $(A, B) T$ is expressed throughout the entire A-P extent of the primitive streak ectoderm and mesoderm, and in the node and notochord of wild-type 2-somite-stage embryos. $(C, D) T$ mRNA is absent in the anterior primitive streak of equivalent stage $W n t 3 a^{-/-}$embryos (arrows), but expression is unaffected in the posterior primitive streak, node, and notochord. $(E, F)$ Tbx6 is expressed throughout the entire A-P extent of the primitive streak and paraxial presomitic mesoderm of wild-type embryos. $(G, H)$ Unlike $T$, Tbx6 continues to be expressed in the anterior primitive streak of early somite stage embryos lacking Wnt3a. $(I, J)$ After the 6-somite stage, $T$ is significantly down-regulated throughout the entire primitive streak in Wnt $3 a$ mutants. In contrast, normal expression levels are observed in the notochord. $(K, L)$ Only a small domain of Tbx6 expression is detected in the posterior-most end of the primitive streak of $W n t 3 a^{-/-}$embryos around the 6 somite stage, whereas strong expression is found throughout the primitive streak and paraxial mesoderm of the wild-type control. 
down-regulation of $T$ in the region of the streak fated to give rise to somites is consistent with the view that $T$ is a target of $W n t 3 a$ signaling during the regulation of paraxial mesoderm fates.

In contrast to T, Tbx6 continued to be expressed strongly in the anterior streak of Wnt3a mutant embryos at the 0 - to 2-somite stage (data not shown) and only moderately down-regulated in Wnt $3 a$ mutant embryos at the 2- to 4-somite stage (Fig. 2G,H). These results suggest that $T$, and not $T b x 6$, may be a direct target of Wnt3a signaling. By the 6-somite stage, low-level $T$ expression was observed in the streak ectoderm of Wnt $3 a^{-1-}$ mutants but not in migrating mesodermal precursors (Fig. 2I,J). Consistent with this observation, Tbx6, which at this time is expressed almost exclusively in migrating mesodermal cells, was virtually absent from the primitive streak (Fig. 2K,L). T expression in the notochord remained unaffected. Thus, in the absence of Wnt $3 a$, early $T$ expression was lost specifically in the anterior primitive streak in the cells normally fated to give rise to paraxial mesoderm. This was followed by a more general reduction of $T b x 6$ and $T$ expression in the mesoderm of the primitive streak, presumably reflecting the widespread loss of mesoderm progenitors at these later stages.

To address the possibility that $T$ is a direct target of Wnt3a signaling, we examined the transcriptional regulation of $T$. It is now well established that a subclass of Wnt family members regulate target gene expression through the nuclear translocation of a transcriptional complex containing $\beta$-catenin and a member of the Tcf family (Nusse 1999). Although Lef1 and Tcf1 single mutants form normal somites, double mutants display a paraxial mesoderm phenotype identical to that observed in Wnt3a mutants, indicating that these factors have redundant roles in transducing Wnt3a signals (Galceran et al. 1999). Both Lef1 and Tcf1 were expressed in the primitive streak of Wnt3a mutants in a normal fashion at 7.5 $\mathrm{dpc}$ and early somite stages, indicating that their expression is independent of Wnt3a (data not shown).

To establish whether $T$ is a direct target of Lef1 and Tcf1, we examined the $T$ promoter for Lef1/Tcf1 (hereafter referred to as Tcf) binding sites. Examination of a proximal 500-bp region of the mouse $T$ promoter that contains elements that are sufficient to drive expression of a reporter gene in the primitive streak domain, but not in the node and notochord (Fig. 3C; Clements et al. 1996), identified two canonical Tcf1 binding sites (Fig. 3A; van de Wetering et al. 1991). A distal site was located at position -358 to -352 , relative to the start of transcription, and a proximal site, in reverse orientation, was located at position -191 to -185 (Fig. 3A). The two Tcf binding sites found in the $T$ promoter are identical to sites found in several vertebrate and invertebrate gene regulatory elements that are known to be Wnt-responsive and capable of binding Lef1 or Tcf1 with high affinity, and in a sequence-specific fashion (Tetsu and McCormick 1999 and references therein). The presence of putative Tcf binding sites is consistent with direct regulation of $T$ by Wnt3a signaling. Interestingly, consensus
A
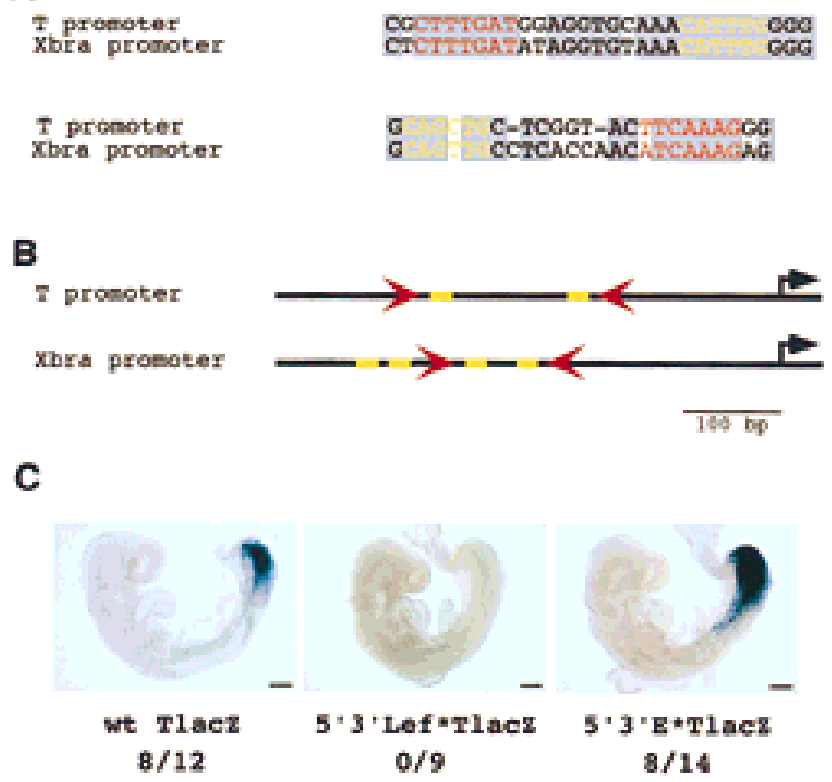

Figure 3. Canonical Tcf binding sites in the $T$ promoter are required for expression in the primitive streak and paraxial mesoderm of transgenic embryos. (A) Two canonical Tcf binding sites (red) are present in the mouse $T$ promoter and conserved in the Xenopus Xbra promoter. A canonical E box motif (yellow) flanks each of the Tcf sites in both the Xenopus and mouse $T$ promoters. $(B)$ A schematic diagram illustrating the relative orientation of the Tcf binding sites (red arrowheads) and the E boxes (yellow rectangles) in the mouse and frog $T$ promoters. $(C)$ (Left) The expression of a lacZ reporter driven by the wild-type $T$ promoter in the primitive streak and paraxial presomitic mesoderm of a transgenic 8.75 dpc embryo; (middle) a representative example of an embryo carrying a $T$ promoter transgene mutated in each of the two Tcf binding sites; (right) an embryo carrying a $T$ promoter transgene mutated in each of the two putative $\mathrm{E}$ boxes. The fraction of embryos expressing the lac $\mathrm{Z}$ transgene in the primitive streak is indicated below. Bar, 200 $\mu \mathrm{m}$.

E boxes presumably capable of binding bHLH transcription factors were also found in the $T$ promoter adjacent to each of these two Tcf binding sites, approximately one turn of the DNA helix away from the adjacent Tcf binding site. The same relative orientation of Tcf binding sites and E boxes was found in the proximal $225 \mathrm{bp}$ of the Xbra (the Xenopus T homolog), and Xbra2 (a pseudogene of Xbra) promoters (Fig. 3A,B; Artinger et al. 1997; Latinkic et al. 1997), which demonstrates a conservation within the regulatory region between these vertebrates, suggesting that these sequences may participate in the assembly of multiprotein enhancer complexes.

To test the model, we first generated mutations in both of the Tcf sites and examined the activity of a reporter gene in transgenic embryos. Eight of 12 (66.6\%) embryos transgenic for the wild-type $T$ promoter expressed the lac $Z$ reporter in the $8.75 \mathrm{dpc}$ primitive streak and hindgut (Fig. 3C, left). The remaining four transgenic embryos had no $\beta$-galactosidase activity at all. Interestingly, no $\beta$-galactosidase activity was detected in the primitive streaks of any of nine independent transgenic 
embryos carrying the mutated transgene (Fig. 3C, middle). These results demonstrate that the Tcf sites are essential for expression of $T$ in the primitive streak. The distal Tcf site alone, or both sites together, is required for activity, as a 95-bp deletion that removes the distal site abolishes promoter activity (Clements et al. 1996). In contrast, a transgene containing mutations generated in both $\mathrm{E}$ boxes of the $T$ promoter was expressed in 8/14 $(57 \%$ ) transgenic embryos (Fig. 3C, right), a similar percentage to the unmodified construct. Thus, despite their position and conservation, the E boxes are not necessary for expression of $T$ in the primitive streak. From these results we conclude that regional expression of $T$ in the primitive streak is most likely directly regulated by signaling through the Wnt3a pathway. Furthermore, this regulatory interaction is essential for the development of most paraxial mesoderm.

Although we have established that $T$ is a target of the Wnt3a pathway, we were interested in determining whether Wnt3a itself could be a target of $T$, thereby establishing an autoregulatory positive feedback loop essential for paraxial mesoderm development. Previous reports have documented that activation of Wnt $3 a$ transcription does not appear to require $T$ activity, as Wnt3a expression is initially normal in the streak of $T$ mutants (Rashbass et al. 1994). Furthermore, Wnt3a transcripts continue to be expressed in Wnt3a mutants in the anterior primitive streak of early somite stage embryos when $T$ is no longer expressed (data not shown). Together, these results indicate that Wnt3a is not a target of $T$; however, the overlapping expression of other T-box-encoding genes with $T$ during gastrulation, such as $T b x 6$ (Chapman et al. 1996), complicates this analysis. $T^{\text {Wis }}$ mutants, which arose through the insertion of a transposable element into the Tlocus (Shedlovsky et al. 1988; Herrmann et al. 1990), have a more severe phenotype than $T$ mutants even though these mutants carry a deletion of the $T$ gene that completely removes activity (Herrmann et al. 1990). $T^{\text {Wis }}$ homozygotes lack somites completely, whereas $T$ homozygotes form 7 somites. These data suggest that the $T^{\text {Wis }}$ allele may dominantly inhibit the activity of other T-box members (Herrmann 1991). Interestingly, we observed significantly reduced Wnt3a expression in the primitive streak of $T^{\text {Wis }} / T^{\text {Wis }}$ embryos at the 0 - to 2-somite stage (Fig. 4D,F; data not shown). In contrast, the loss of Wnt3a transcripts observed in $T / T$ homozygotes was not observed until the 4to 5-somite stage (Rashbass et al. 1994). The correlation between the stage at which Wnt3a transcription is down-regulated and the severity of the $T$ allele suggests that Wnt3a may be regulated by T-box activity. Consistent with this hypothesis, examination of Wnt3a homozygotes at later stages (5-6 somites) reveals that both Wnt3a (Fig. 4A,B), and T (Fig. 2J) are coexpressed weakly in the mutant streak. Proof of a reciprocal autoregulatory loop that maintains both $T$ and $W n t 3 a$ expression in the streak will require a thorough characterization of the $W n t 3 a$ regulatory region and the analysis of compound mutants in T-box genes.

In conclusion, we have demonstrated that $W n t 3 a$ regu-

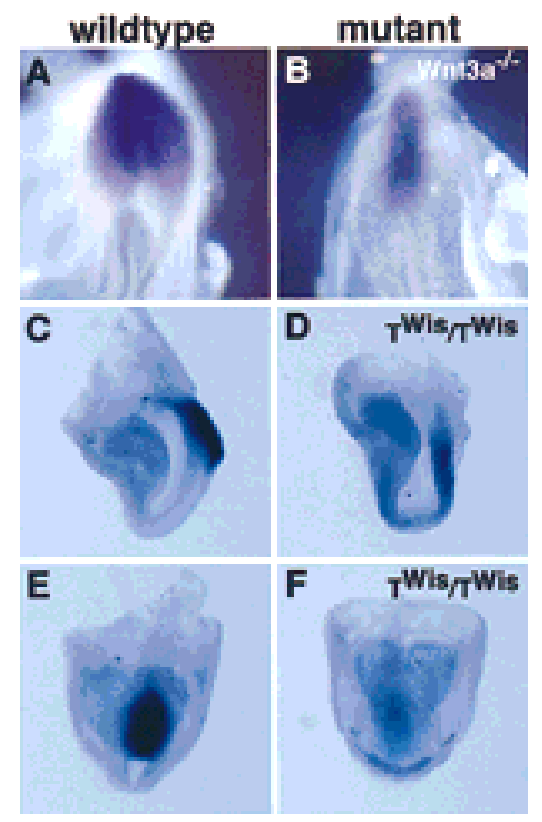

Figure 4. Analysis of Wnt3a expression in Wnt $3 a^{-/-}$and $T^{\text {Wis }}$ mutant embryos. Dorsal views of the primitive streak region of 5- to 6-somite stage wild-type $(A)$ and $W n t 3 a^{-/-}(B)$ embryos. Lateral views of $W n t 3 a$ expression in the primitive streak of a 3 -somite wild-type $(C)$ and the equivalent of a 2-somite $T^{\text {Wis }}$ homozygote (staging based on headfold morphology) $(D)$. The reduced expression of $W n t 3 a$ in the $T^{W i s}$ mutant primitive streak $(F)$ compared to the wild-type $(E)$ is more evident in a ventral-posterior view of the same embryos depicted in $C$ and $D$.

lates paraxial mesoderm development in the anterior primitive streak, at least in part, through the direct regulation of the mesodermal determinant T. Analyses of the $W n t 3 a$ and $T$ mutant phenotypes demonstrate that specification of mesodermal cell fates and extension of the A-P body axis are intimately linked processes during embryogenesis. Animals heterozygous for null alleles of either Wnt3a or T display kinked or shortened tail phenotypes due to haploinsufficiency (Dobrovolskaia-Zavadskaia 1927; Greco et al. 1996). Examination of ordered allelic series of mutations in either Wnt $3 a$ or $T$ demonstrates a correlation between the severity of the axial truncation and gene dosage (MacMurray and Shin 1988; Greco et al. 1996) and suggests that Brachyury and Wnt3a participate in the development of the entire A-P axis. Our demonstration that $T$ is a transcriptional target of the Wnt3a signaling pathway is consistent with this genetic data and suggest that a primary function of $W n t 3 a$ may be to tightly regulate the dosage of $T$ during embryogenesis.

In wild-type embryos, pluripotent epithelial epiblast cells of the anterior primitive streak ingress through the streak and become mesenchymal upon exposure to mesoderm-promoting factors such as Wnt $3 a$. In the absence of either $W n t 3 a$ or $T$, epiblast cells retain their epithelial character and assume a neural fate. Nevertheless, these putative neural epithelial cells continue to ingress through the primitive streak to form an ectopic tube that ultimately lies ventral to the primary neural tube. Thus, 
continued expression of Wnt $3 a$ and $T$ is essential for mesodermal progenitors to adopt specific mesodermal fates and to promote mesenchymal morphology but neither gene appears essential for ingression of cells through the streak. How exactly Wnt3a and Brachyury control these cellular processes is currently unclear.

One demonstrated function for $T$ is in the regulation of morphogenetic cell movements (Wilson et al. 1995). Experimentally increasing the levels of Brachyury in streak cells led to increased movement of these cells away from the primitive streak (Wilson and Beddington 1997). Interestingly, different cells in the wild-type streak appear to express different levels of Brachyury (Kispert and Herrmann 1994; Wilson and Beddington 1997). Because we have demonstrated that $W n t 3 a$ regulates $T$ transcription directly, it seems likely that Wnt3a modulates the proportion of cells that stay in the streak versus cells that exit the streak as paraxial mesoderm progenitors. It is tempting to speculate that Wnt3a specifies the fate of a pluripotent primitive streak stem cell (Tam and Beddington 1987; Nicolas et al. 1996; Wilson and Beddington 1996) to give rise to a paraxial mesodermal daughter cell that expresses high levels of $T$ and exits the streak to contribute to trunk somites. The suggestion has been made from amphibian studies that neural development is a default state, which, in contrast to mesoderm development, does not require a specific inductive process. Our results here are consistent with this general model in that Wnt3a is required for paraxial mesoderm development; its absence leads to ectopic neural development. Thus, Wnt3a ultimately modulates a balance between mesodermal and neural cell fates in the primitive streak during gastrulation and A-P axis development.

\section{Materials and methods}

\section{Embryo collection and analysis}

Embryos were dissected into PBS and yolk sacs were biopsied for PCR genotyping. The targeted Wnt $3 a$ allele was genotyped by PCR as described (Takada et al. 1994). Embryos homozygous for $T^{\text {Wis }}$ were generated by intercrossing $T^{W i s}$ heterozygotes (easily identified by their lack of tails) and were identified morphologically by their lack of somites. Homozygous $T$ embryos were also generated by intercrossing $T$ heterozygotes. Experiments performed on $T$ and $T^{W i s}$ homozygous embryos were repeated four times. Embryos were fixed in $4 \%$ paraformaldehyde for whole-mount RNA in situ hybridization analysis according to the method of Wilkinson and Nieto (1993). Embryos dissected for $\beta$-galactosidase staining were fixed in $1 \%$ formaldehyde $/ 0.2 \%$ glutaraldehyde and processed following the method of Whiting et al. (1991). Whole-mount stained embryos were processed for paraffin embedding and sectioned as described (Takada et al. 1994; Yoshikawa et al. 1997).

\section{Transgene construction}

A $14.7-\mathrm{kb}$ plasmid (pCTZA) containing $8.3 \mathrm{~kb}$ of the murine $T$ promoter fused to lacZ and followed by an SV40 polyadenylation sequence (Stott et al. 1993) was cut with SacII and SalI and subcloned into pBluescript KS(+) (Stratagene). This plasmid was designated pBS0.7TZA and retained $\sim 500$ bp of the proximal $T$ promoter fused to the lac $Z$ reporter. pBS0.7TZA served as the wild-type $T$ promoter control construct and has been shown to contain elements sufficient to drive expression in the primitive streak (Clements et al. 1996). The promoter was isolated further with BamHI and SacII and subcloned into pBluescript to generate $\mathrm{pBSO} .7 \mathrm{~T}$; this promoter construct served as the template for subsequent mutagenesis. Mutagenized promoters were cloned into $\mathrm{pBS} 0.7 \mathrm{TZ}(\mathrm{S}-\mathrm{C})$, a shuttle vector created by subcloning a 1.4-kb SacII-ClaI fragment that contained the $500-$ bp promoter and a portion of lacZ into pBluescript. The lacZ reporter was reconstituted by cloning the 1.4-kb fragment containing the mutagenized promoter back into pBS0.7TZA.

The $T$ promoter was mutagenized following the QuikChange site-directed mutagenesis kit (Stratagene) protocol. The 5' and 3' Tcf binding sites were mutagenized using the following primers: $2 \mathrm{~F}-5^{\prime}{ }^{\prime}{ }^{*}{ }^{\star}$, CCAGGGTCCGCCCCGCCGCGAATTCGGAGGTGCAAACATTTGG; 2R-5' Lef $^{*}$, CCAAATGTTTGCACCTCCGAATTCGCGGCGGGGCGGACCCTGG; 3F-3'Lef*, GGGCAGCTGCTCGGTACTTCCCCGGGTGTCCCGCCCAATCCGCC; and 3R-3'Lef*, GGCGGATTGGGCGGGACACCCGGGGAAGTACCGAGCAGCTGCCC. The 5' and $3^{\prime}$ E boxes were mutagenized using the following primers: $2 \mathrm{~F}-5^{\prime} \mathrm{E}^{\star}$, GCGAATTCGGAGGTGCAAAGAAGCTTGGGAGGGCGGGGGTGTCGGG; 2R-5'E* , CCCGACACCCCCGCCСTCCCAAGCTTCTTTGCACCTCCGAATTCGC; 2F-3'E* , GGCCGCGCACCGCCAATGGGTGGCCACTCGGTACTTCCCCGGGTG; 2R-3'E* ${ }^{\star}$ CACCCGGGGAAGTACCGAGTGGCCACCCATTGGCGGTGCGCGGCC. All primers were synthesized by Life Technologies and PAGE-purified. All PCR mutagenesis reactions were performed using 18 cycles of denaturing at $95^{\circ} \mathrm{C}$ for $30 \mathrm{sec}$, annealing at $55^{\circ} \mathrm{C}$ for $1 \mathrm{~min}$, followed by an 8 -min extension at $68^{\circ} \mathrm{C}$.

Generation of transgenic embryos

DNA constructs were prepared for pronuclear injection by digesting with SacII and SalI and purifying by agarose gel to remove vector sequences. DNA was electroeluted in dialysis tubing in $1 \times \mathrm{TAE}$ and purified using several QIAQuick PCR DNA purification spin columns (Qiagen). Eluants were pooled and ethanol precipitated, and DNA was resuspended in $50 \mu \mathrm{l}$ of TE at $\mathrm{pH}$ 7.5. Concentration was determined by UV spectrophotometry and confirmed by gel electrophoresis using known standards.

Pronuclear injections were performed according to standard published protocols (Hogan et al. 1994). G0 transgenic embryos ( $8.5 \mathrm{dpc}$ ) were dissected and yolk sacs were biopsied for PCR genotyping using primers to the $T$ promoter $[T(+7 /+20)$ : CCTTTGGCGAATGTGCAGGG] and to lacZ (oligo 1098: AAGGGCGATCGGTGCGGGCC). Samples were cycled $35 \times$ at $94^{\circ} \mathrm{C}$ for $30 \mathrm{sec}, 66^{\circ} \mathrm{C}$ for $30 \mathrm{sec}$, and $72^{\circ} \mathrm{C}$ for $1 \mathrm{~min}$.

\section{Acknowledgments}

We thank D. Stott for kindly providing the $T$ promoter plasmid pCTZA, and D. Chapman and V. Papiannou for the Tbx6 probe. We also thank E. Robertson for providing $T^{W i s}$ mice. T.P.Y. acknowledges the kind support of the Human Frontiers Science Program (HFSP) and the Medical Research Council of Canada. Work in S.T.'s laboratory was supported by grants from the the Ministry of Education, Science, and Culture of Japan, JST, Takeda Science Foundation, and Kato Memorial Bioscience Foundation. Work in A.P.M.'s laboratory was supported by a grant from the National Institutes of Health/National Institute of Child Health and Human Development (HD30249).

The publication costs of this article were defrayed in part by payment of page charges. This article must therefore be hereby marked "advertisement" in accordance with 18 USC section 1734 solely to indicate this fact.

\section{References}

Artinger, M., I. Blitz, K. Inoue, U. Tran, and K.W. Cho. 1997. Interaction of goosecoid and brachyury in Xenopus mesoderm patterning. Mech. Dev. 65: 187-196.

Cadigan, K.M. and R. Nusse. 1997. Wnt signaling: A common theme in animal development. Genes \& Dev. 11: 3286-3305.

Chapman, D.L. and V.E. Papaioannou. 1998. Three neural tubes in mouse embryos with mutations in the T-box gene Tbx6. Nature 391: 695697.

Chapman, D.L., I. Agulnik, S. Hancock, L.M. Silver, and V.E. Papaioannou. 1996. Tbx6, a mouse T-Box gene implicated in paraxial mesoderm formation at gastrulation. Dev. Biol. 180: 534-542.

Chesley, P. 1935. Development of the short-tailed mutant in the house mouse. J. Exp. Zool. 70: 429-435.

Clements, D., H.C. Taylor, B.G. Herrmann, and D. Stott. 1996. Distinct regulatory control of the Brachyury gene in axial and non-axial mesoderm suggests separation of mesoderm lineages early in mouse gastrulation. Mech. Dev. 56: 139-149.

Dobrovolskaia-Zavadskaia, N. 1927. Sur la mortification sponta-nee de la queue chez la souris nouveau-nee et sur l'existence d'un caractere hereditaire "non viable." C. R. Hebd. Soc. Biol. 97: 114-116. 
Galceran, J., I. Farinas, M.J. Depew, H. Clevers, and R. Grosschedl. 1999. Wnt $3 \mathrm{a}^{-/-}$-like phenotype and limb deficiency in Lef1 ${ }^{-/-} \mathrm{Tcf}^{-/-}$mice. Genes \& Dev. 13: 709-717.

Greco, T.L., S. Takada, M.M. Newhouse, J.A. McMahon, A.P. McMahon, and S.A. Camper. 1996. Analysis of the vestigial tail mutation demonstrates that Wnt-3a gene dosage regulates mouse axial development. Genes \& Dev. 10: 313-324.

Herrmann, B.G. 1991. Expression pattern of the Brachyury gene in wholemount TWis/TWis mutant embryos. Development 113: 913-917.

Herrmann, B.G., S. Labeit, A. Poustka, T.R. King, and H. Lehrach. 1990 Cloning of the $\mathrm{T}$ gene required in mesoderm formation in the mouse. Nature 343: 617-622.

Hogan, B., R. Beddington, F. Constantini, and E. Lacy. 1994. Manipulating the mouse embryo, a laboratory manual. Cold Spring Harbor Laboratory Press, Cold Spring Harbor, NY.

Kispert, A. and B.G. Herrmann. 1994. Immunohistochemical analysis of the Brachyury protein in wild-type and mutant mouse embryos. Dev. Biol. 161: 179-193.

Latinkic, B.V., M. Umbhauer, K.A. Neal, W. Lerchner, J.C. Smith, and V. Cunliffe. 1997. The Xenopus Brachyury promoter is activated by FGF and low concentrations of activin and suppressed by high concentrations of activin and by paired-type homeodomain proteins. Genes \& Dev. 11: 3265-3276.

MacMurray, A. and H.-S. Shin. 1988. The antimorphic nature of the $T^{c}$ allele at the mouse $T$ locus. Genetics 120: 545-550.

Moon, R., J. Brown, and M. Torres. 1997. WNTs modulate cell fate and behavior during vertebrate development. Trends Genet. 13: 157-162.

Nicolas, J.F., L. Mathis, C. Bonnerot, and W. Saurin. 1996. Evidence in the mouse for self-renewing stem cells in the formation of a segmented longitudinal structure, the myotome. Development 122: 2933-2946.

Nusse, R. 1999. WNT targets. Repression and activation. Trends Genet. 15: 1-3.

Rashbass, P., V. Wilson, B. Rosen, and R.S. Beddington. 1994. Alterations in gene expression during mesoderm formation and axial patterning in Brachyury (T) embryos. Int. J. Dev. Biol. 38: 35-44.

Shedlovsky, A., T.R. King, and W.F. Dove. 1988. Saturation germ line mutagenesis of the murine $t$ region including a lethal allele at the quaking locus. Proc. Natl. Acad. Sci. 85: 180-184.

Smith, J. 1999. T-box genes: What they do and how they do it. Trends Genet. 15: 154-158.

Stott, D., A. Kispert, and B.G. Herrmann. 1993. Rescue of the tail defect of Brachyury mice. Genes \& Dev. 7: 197-203.

Takada, S., K.L. Stark, M.J. Shea, G. Vassileva, J.A. McMahon, and A.P. McMahon. 1994. Wnt-3a regulates somite and tailbud formation in the mouse embryo. Genes \& Dev. 8: 174-189.

Tam, P.P. and R.S. Beddington. 1987. The formation of mesodermal tissues in the mouse embryo during gastrulation and early organogenesis. Development 99: 109-126.

Tam, P.P. and P.A. Trainor. 1994. Specification and segmentation of the paraxial mesoderm. Anat. Embryol. 189: 275-305.

Tetsu, O. and F. McCormick. 1999. Beta-catenin regulates expression of cyclin D1 in colon carcinoma cells. Nature 398: 422-426.

van de Wetering, M., M. Oosterwegel, D. Dooijes, and H. Clevers. 1991. Identification and cloning of TCF-1, a T lymphocyte-specific transcription factor containing a sequence-specific HMG box. EMBO $T$. 10: $123-132$.

Whiting, J., H. Marshall, M. Cook, R. Krumlauf, P.W. Rigby, D. Stott, and R.K. Allemann. 1991. Multiple spatially specific enhancers are required to reconstruct the pattern of Hox-2.6 gene expression. Genes \& Dev. 5: 2048-2059.

Wilkinson, D.G. and M.A. Nieto. 1993. Detection of messenger RNA by in situ hybridization to tissue sections and whole mounts. Methods Enzymol. 225: 361-373.

Wilkinson, D.G., S. Bhatt, and B.G. Herrmann. 1990. Expression pattern of the mouse $\mathrm{T}$ gene and its role in mesoderm formation. Nature 343: 657-659.

Wilson, V. and R.S. Beddington. 1996. Cell fate and morphogenetic movement in the late mouse primitive streak. Mech. Dev. 55: 79-89.

- 1997. Expression of T protein in the primitive streak is necessary and sufficient for posterior mesoderm movement and somite differentiation. Dev. Biol. 192: 45-58.

Wilson, V., L. Manson, W.C. Skarnes, and R.S. Beddington. 1995. The T gene is necessary for normal mesodermal morphogenetic cell movements during gastrulation. Development 121: 877-886.

Yoshikawa, Y., T. Fujimori, A.P. McMahon, and S. Takada. 1997. Evidence that absence of Wnt-3a signaling promotes neuralization in stead of paraxial mesoderm development in the mouse. Dev. Biol. 183: $234-242$. 


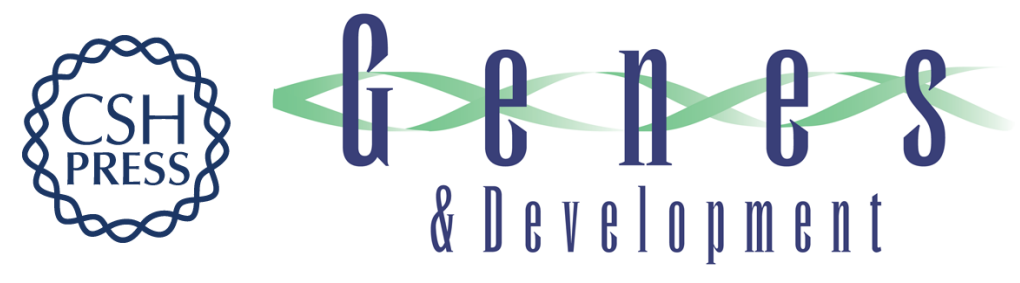

\section{$T$ (Brachyury) is a direct target of Wnt3a during paraxial mesoderm specification}

Terry P. Yamaguchi, Shinji Takada, Yoshiaki Yoshikawa, et al.

Genes Dev. 1999, 13:

References This article cites 32 articles, 13 of which can be accessed free at:

http://genesdev.cshlp.org/content/13/24/3185.full.html\#ref-list-1

License

Email Alerting Receive free email alerts when new articles cite this article - sign up in the box at the top Service right corner of the article or click here.

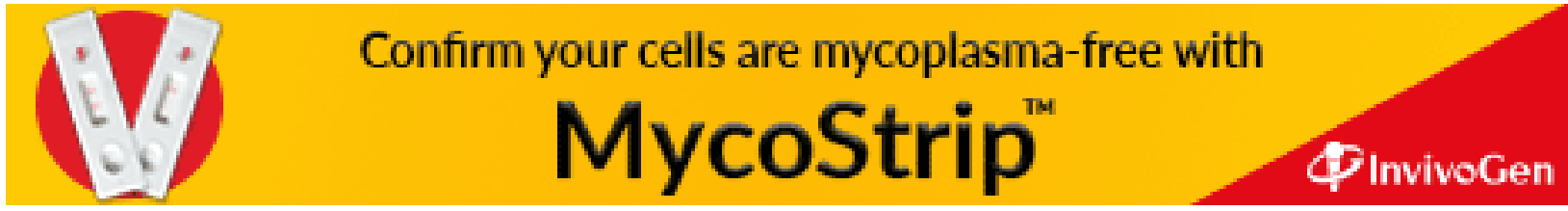

